Military Technical College Kobry El-Kobbah, Cairo, Egypt

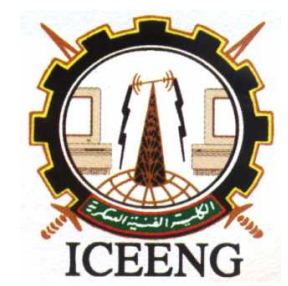

\author{
$6^{\text {th }}$ International Conference \\ on Electrical Engineering \\ ICEENG 2008
}

\title{
Fabrication and simulation of optimized loaded helical antennas
}

$$
\text { By }
$$

Ahmed Ibrahim Salem *

\section{Abstract:}

In this paper the helical antenna is considered with different helix diameters; uniform, linear, conical and exponential diameter variations are loaded at different positions. All helices are mounted on infinite ground planes and analyzed using NEC commercial code based on the moment methods. In each case, the radiation fields in the principal planes, the gains, axial ratio and current distributions are obtained. Also the input impedance is calculated as the frequency varies around the design values for each case. In all cases the pitch is taken constant. The numerical evaluations indicate that the conical helix gives better performance. Fabrication of uniform and conical helices is accomplished by their accurate dimensions .The results clarify that the laboratory measured values is very close to the computed results.

\section{Keywords:}

Helical antenna, NEC commercial code. 


\section{Introduction:}

When connecting an antenna to a transmission line it is important to make effective use of all available power from the transmitter in the transmit case and from the antenna in the receive case [1]. There are two primary considerations: the impedance match between the antenna and transmission line, and the excitation of the current distribution on the antenna. In this paper, the input part of the helical antennas is put under inspection [2]. Design of uniform and conical helices is achieved. Trials for increasing in the performance by adding resistive and inductive loads are done.

NEC [3] offers us short-cut methods of modeling both solenoid and the stub reactive loads via purely mathematical models. Before we conclude this sequence of notes, we shall investigate modeling both types of reactive loads, linear or transmission line loads and solenoid loads as physical wire assemblies. One reason for taking pains to develop the models used in the preliminary investigation of using the LD and TL functions of NEC [4] for antenna loads is that physically modeling both transmission line loads and single layer solenoid (or helical) loads requires care lest one exceeds one or another limitation of the NEC modeling calculation system.

Feed point impedance of air wound helix antennas in axial mode is $137 \mathrm{Ohm}$ (almost purely resistive). Matching is required if $50 \mathrm{Ohm}$ coax cable is to be connected. There are two common ways to do this matching. Either with a coax section or a stripline above a ground plane, both a quarter $\lambda$ long and of proper resistance. The "proper resistance" is calculated as follows.

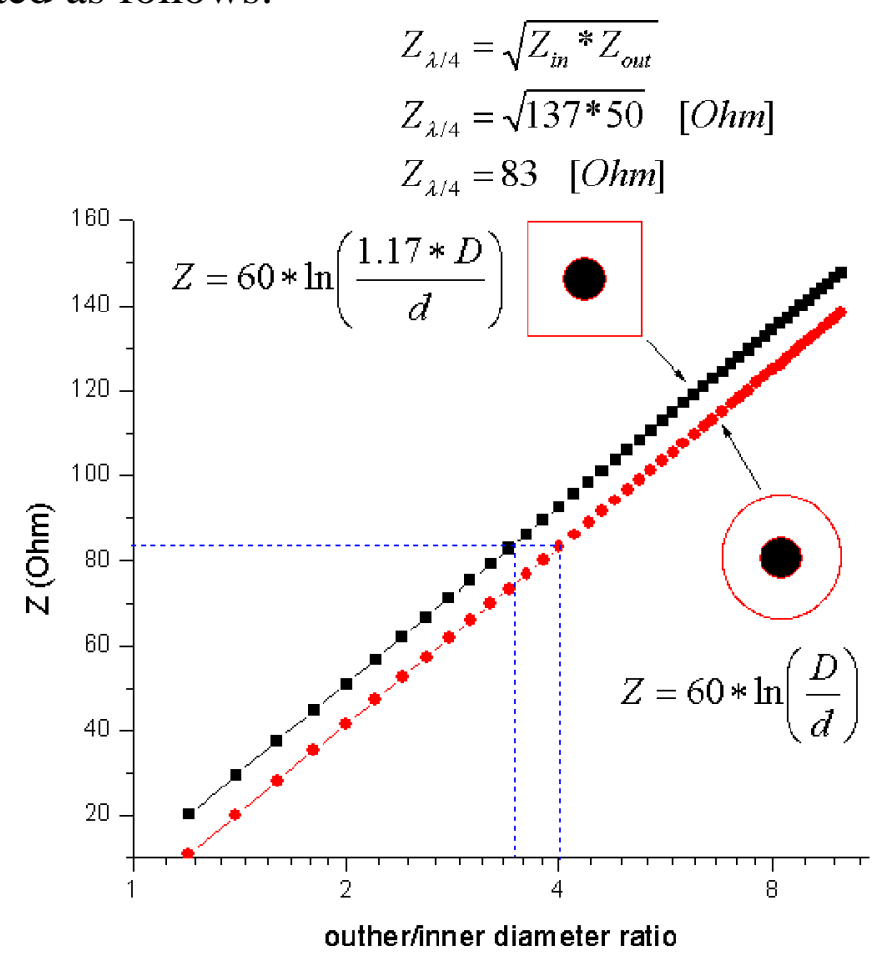

Figure (1) outer to inner diameter ratio calculation 
For an antenna mounted above a ground plane, it is well known that the physical extent of the ground plane has an appreciable effect on the antenna input impedance and radiation characteristics [5]. Conical antennas mounted above ground planes are widely used as they generate a vertically polarized, omnidirectional field and have wideband operating characteristics. Earlier analyses have investigated the radiation properties of conical antennas above infinite ground planes [6] and this paper shows fabrication facility.

\section{FABRICATION OF HELICAL ANTENNA}

From concept to working prototype takes just a few hours. A simplified technique has been applied for fabricating helical antennas for use in experiments on radio-frequency generation. These antennas are typically made of aluminum (for electrical conductivity) and must have a specific helical shape and precise diameter. Such an antenna could be made by bending a single long piece of aluminum or copper tubing or bending smaller pieces of copper tubing, then welding the pieces together. It could also be made by machining from a single large piece of copper. It is extremely difficult to bend copper tubing into a helix with a precise pitch and diameter but it is more easily done using aluminum. It is also difficult to create the helical shape from multiple pieces of tubing; moreover, welding separate pieces distorts the shape. Machining a hollow cylindrical helix from a block or cylinder of copper entails the use of a complex, expensive, threedimensional-milling machine in a process that entails long setup and machining times [7]. In the present simplified technique, one begins by creating a two-dimensional paper template of a desired helical antenna shape. The template is pasted on the outer surface of a copper pipe that has the desired inner and outer diameters. Holes are drilled at the locations where corners are required to exist in the final helical antenna. Manually, using a hacksaw, diagonal cuts are made in the outer cylindrical surface of the pipe, following the lines on the template. Usually, after hacksawing, only a little filing is needed to smooth the edges of the resulting antenna. If the antenna must be watercooled, then copper tubing can be brazed onto the outer surface of the antenna. This tubing is not required to follow the precisely defined shape of the antenna [8]. Our prototype antennas are uniform and conical shaped constructed using aluminum. The helices parameters are computed and tabulated in table 1. This fabrication technique would not be suitable for mass production, but it is ideal for a laboratory environment. The advantages of this technique are the following: (i) Precise antennas can be made from inexpensive, stock-size copper or pipes of aluminum. (ii). No welding of separate pieces is needed, and so there is no welding-induced distortion of antenna shapes.(iii) Prototype antennas can be fabricated fairly rapidly, without the need for complex threedimensional-milling machines or computer-aided drafting tools. (iv) Notwithstanding the reliance on handwork, the total fabrication time (as little as a few hours) is competitive with, and probably less than, that of any automated process that could be used for this purpose. 
TABLE 1

\begin{tabular}{|l|c|c|}
\hline Antenna profile & Uniform & Conical \\
\hline \hline Frequency & \multicolumn{2}{|c|}{$433 \mathrm{MHz}$} \\
\hline Number of Turns & 12 & 6 \\
\hline Length of turn & $76 \mathrm{Cm}$ & Variable conically \\
\hline Spacing Between Turns & 16.5 & $17 \mathrm{Cm}$ \\
\hline Diameter & 25.8 & 36.8 \\
\hline Antenna Length & $200 \mathrm{Cm}$ & $70 \mathrm{Cm}$ \\
\hline Computed Gain & $13.69 \mathrm{~dB}$ & $12 \mathrm{~dB}$ \\
\hline
\end{tabular}

\section{SIMULATION RESULTS}

All helices are simulated at central frequency $433 \mathrm{MHz}$, constant pitch and infinite ground plane applied. Figure (2) shows the gain of the four helix profiles at various lengths $0.4 \lambda, 0.6 \lambda, 0.7 \lambda, 0.9 \lambda, \lambda$, and $1.2 \lambda$ and loaded at different positions of helix. Clear enhancement in the gain performance is noticed when loaded from segment number (160 to 200) at length $\lambda$ for uniform helix, while the worst case is for exponential helix loaded from segment (20 to 60) at small lengths.

Figure (3) investigates the electric field in the vertical plane. The narrower patterns are produced by uniform, linear, and conical helices. Higher front-to-back ratios are got for linear and conical helices at lengths $0.9 \lambda, \lambda$, and $1.2 \lambda$, loaded from segment number (160 to 200), while the lowest front-to-back ratios are for exponential helix. Figure (4) depicts the current distribution for all helices. Optimum values are accomplished for linear helix especially at lengths $\lambda$, and $1.2 \lambda$. The improvement is obvious in the case of conical helix at lengths $0.9 \lambda, \lambda$, and $1.2 \lambda$ when loaded at different positions of the helix.

Figures (5) and (6) clarify the input impedance, resistance and reactance versus frequency. It is obvious that in all cases the input impedance has higher values when loaded at lengths $0.7 \lambda, 0.9 \lambda$ for uniform and linear helices. The improvement in the input reactance is noticed when loaded at segments (160 to 200) for uniform and linear helices.

In Figure (7), the polarization versus angle is computed for the various kinds of helices. It is clear that the directionality increases at lengths $0.6 \lambda$, and $0.7 \lambda$ when loaded from segment number (160 to 200) for conical helix which has the highest axial ratio. Figure (8) show the laboratory photos of the fabricated uniform and conical helices and their feeding and matching schemes. Figures (9) and (10) show the normalized electric field laboratory measured and computed via NEC code for both uniform and conical helices. It is obvious that the measured values have slight variation than the results computed via 
NEC code. This variation is for the high precautions taken in consideration during the design procedure, matching of both antennas. The measured electric field proves successful design, high directionality and high gain for both types of antennas. The conical antenna has a narrower pattern than that measured for the uniform antenna.

\section{CONCLUSION}

A convenient method for the design of uniform, linear, exponential, and conical profiles is considered with constant pitch $0.25 \lambda$ and constant large diameter $0.014 \lambda$. The infinite ground plane is used to get better characteristics. Loading by resistive and inductive loads in different positions of helix are considered and it is obvious that the inductive loading gives better results than resistive one and when loading the helix at the beginning gives higher performance than loading at its end. Also changing the length in terms of $\lambda$ with the change of the loading position in the conical helix gives better performance especially at infinite ground which gives narrower patterns. Also it shows higher front-to-back ratio. It is obvious that the linear helix competes in performance with the conical helix. The electric field is computed in the vertical plane. The gain of each profile is investigated. The polarization versus angle is also computed showing circular polarization for helical profiles, vertical for conical helix. Input impedance real and imaginary is calculated versus frequency. The current distribution is enhanced at the previous constrains. Conical and linear profiles show higher performance than other helices. Practical fabrication of uniform and conical antennas is achieved. Measurements of both types is accomplished and compared by the results computed by the NEC code and both are very close. Design of other types of antennas and their simulation will be studied in the future. 


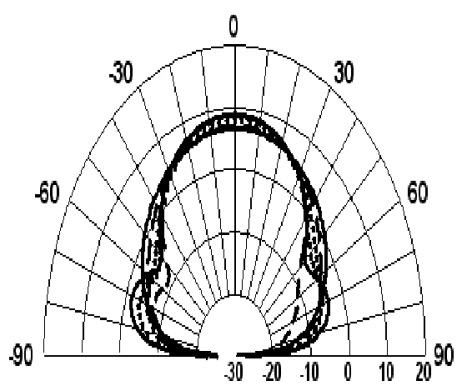

(a) Uniform

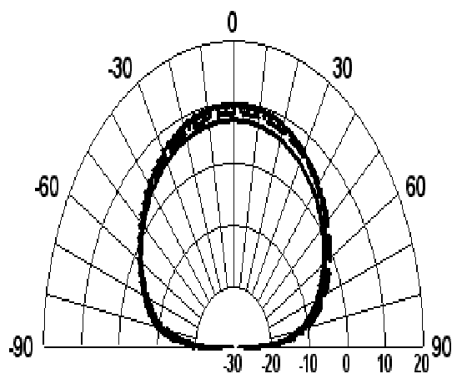

(c) Conical

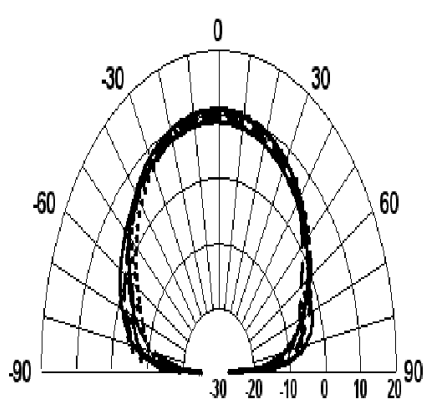

(b) Linear

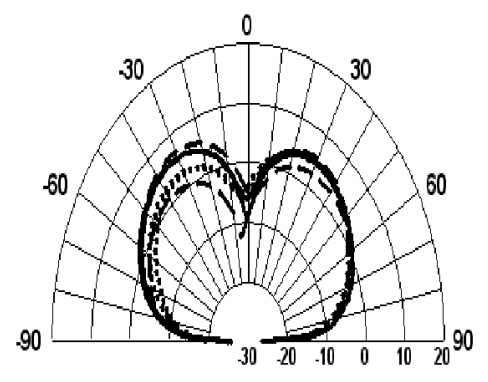

(d) Exponential

Figure (2) The gain for the four profiles at different lengths in terms of $\lambda$ series Loaded by inductance $260 \mathrm{mH}$ at segments 160 to 200

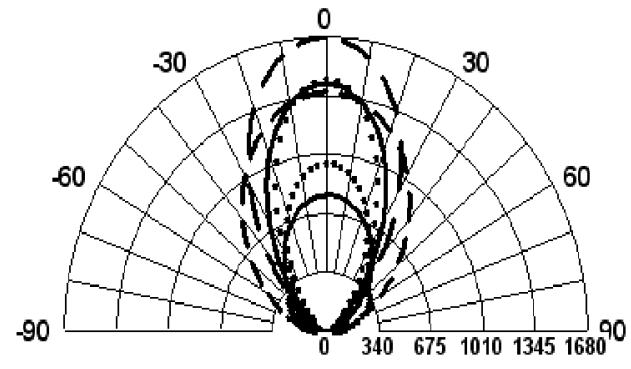

(a) Uniform

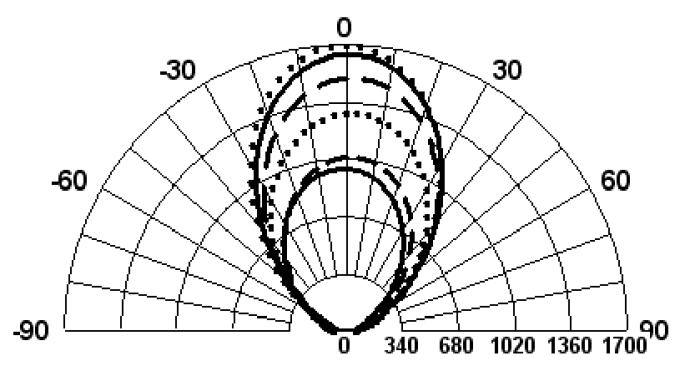

(c) Conical

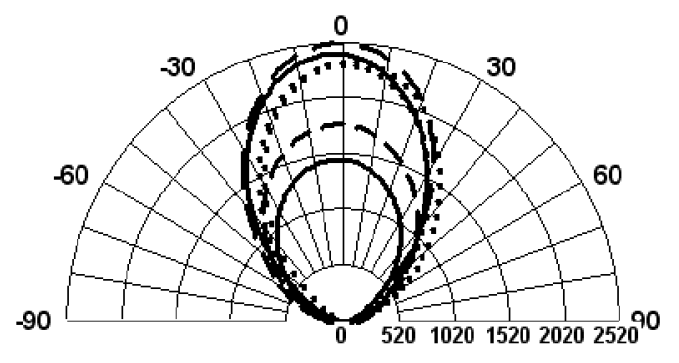

(b) Linear

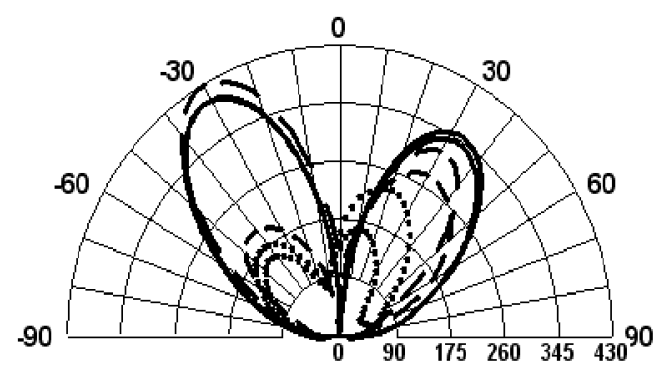

(d) Exponential

Figure (3) Electric field for the four profiles at different lengths in terms of $\lambda$ series Loaded by inductance $260 \mathrm{mH}$ at segments 160 to 200 


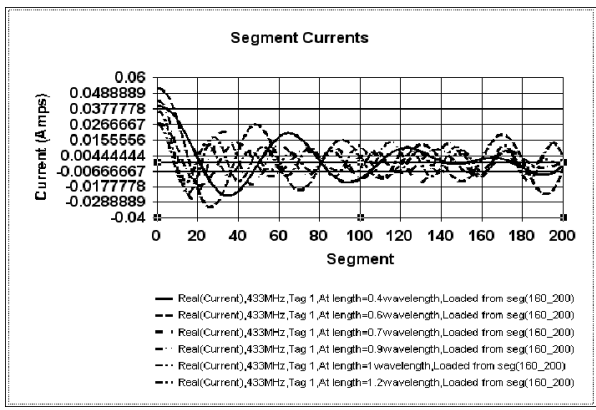

(a) Uniform

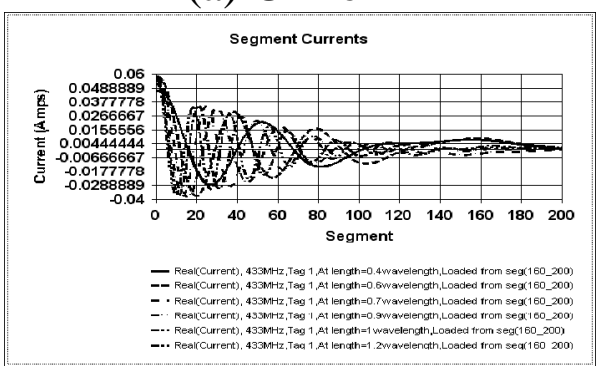

(c) Conical

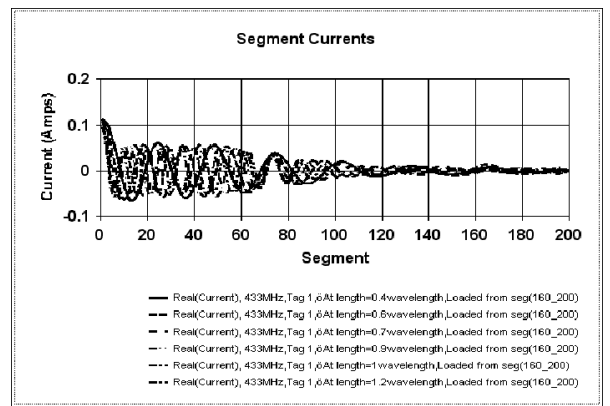

(b) Linear

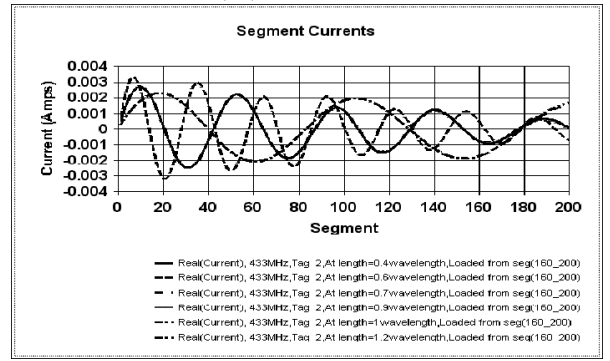

(d) Exponential

Figure (4) The current distribution for the four profiles at different lengths in terms of $\lambda$ series Loaded at segments 160 to 200 by inductance $260 \mathrm{mH}$

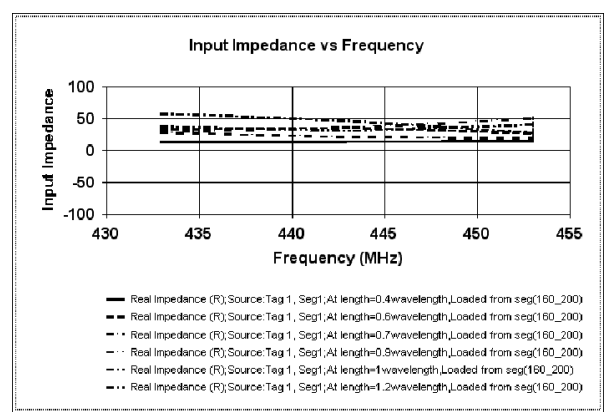

(a) Uniform

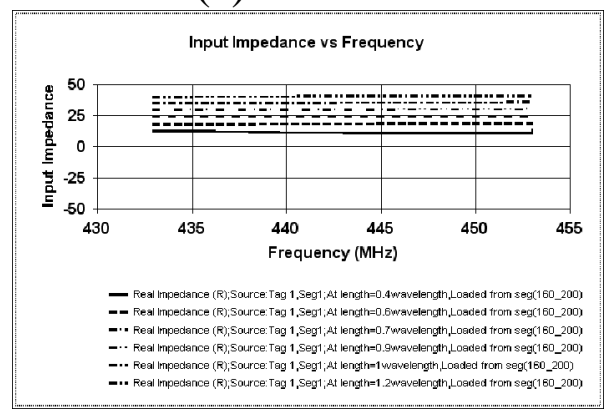

(c) Conical

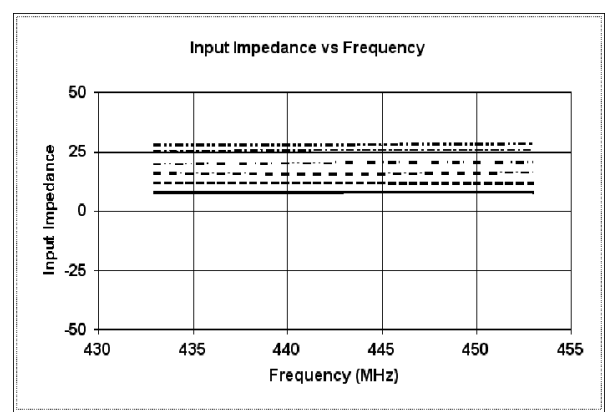

(b) Linear

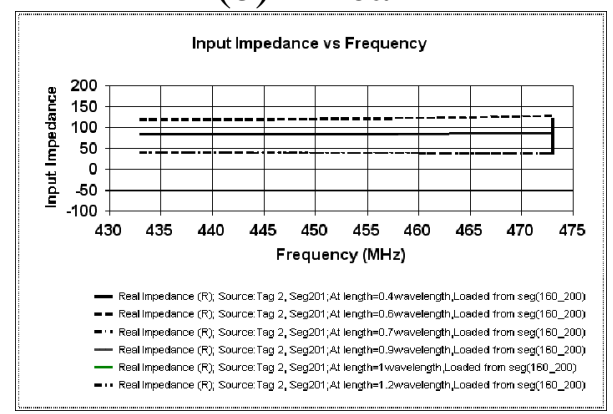

(d) Exponential

Figure (5) The input resistance for the four profiles at different lengths in terms of $\lambda$ series Loaded a by inductance $260 \mathrm{mH}$ 

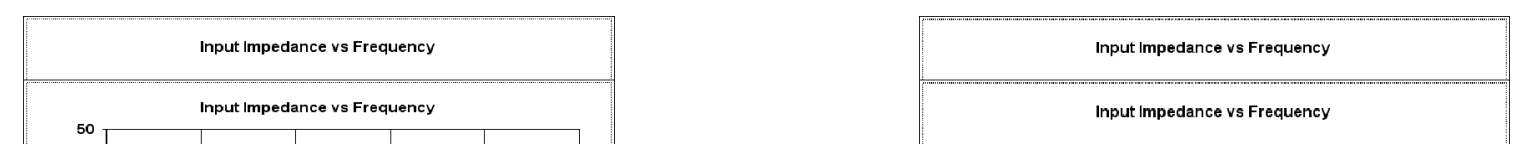

Figure (6) The input reactance for the four profiles at different lengths in terms of $\lambda$ series

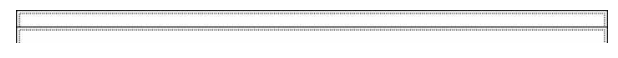




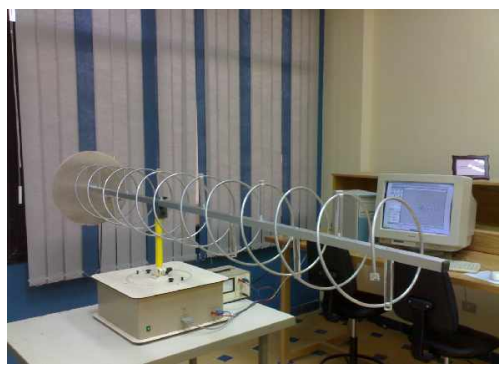

(b) Fabricated Conical Helix

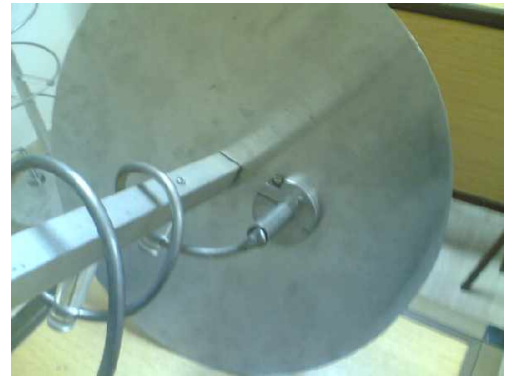

(d) Conical Connector Matching

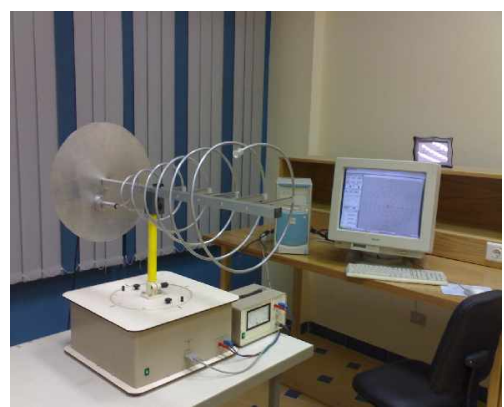

(a) Fabricated Uniform Helix

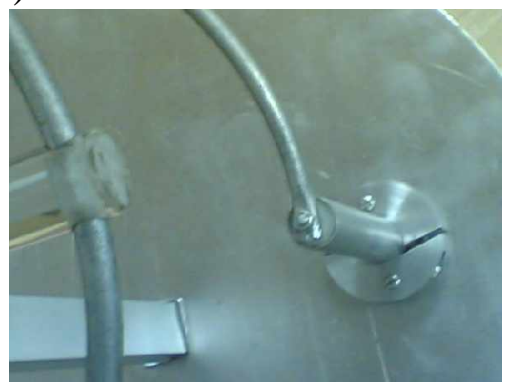

(c) Uniform BNC Connector Figure (8) Uniform and Conical Helices Fabrication

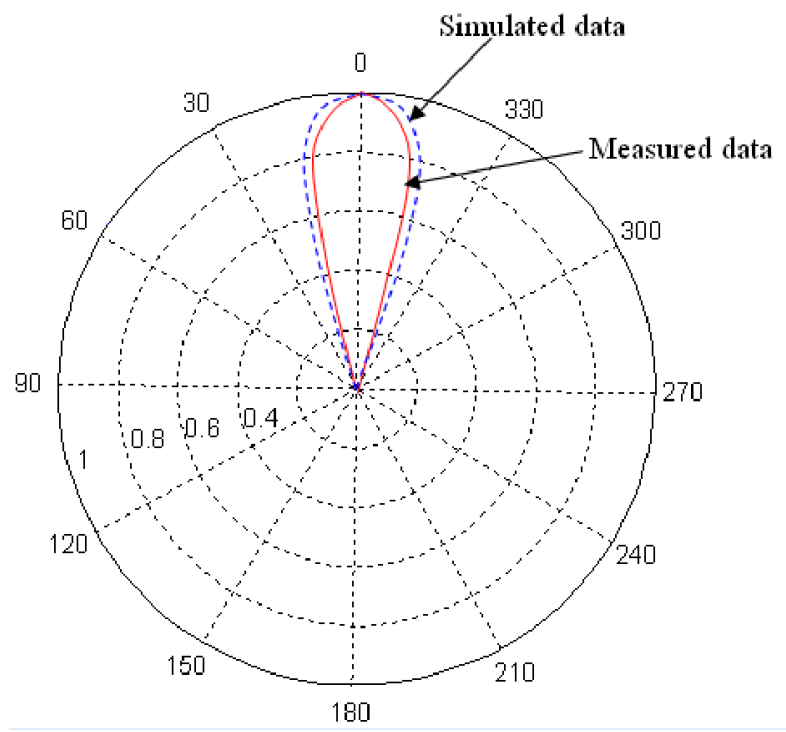

Figure (9) Comparison Between Conical Helix Laboratory Measurements and Results Computed Via NEC code 


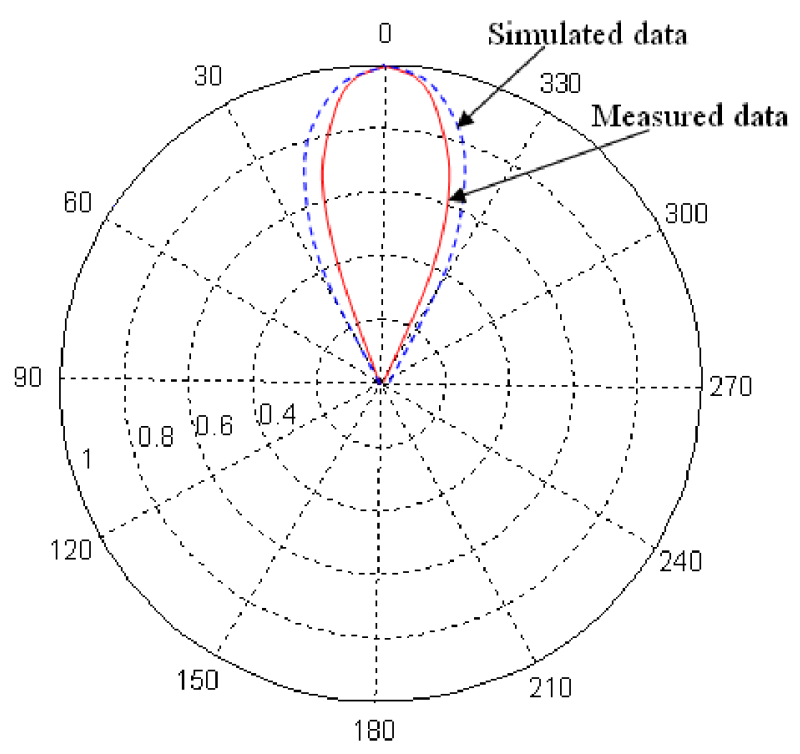

Figure (10) Comparison Between Uniform Helix Laboratory Measurements and Results Computed Via NEC code

\section{$\underline{\text { References }}$}

[1] Kraus, J.D., "Antennas," third edition, McGraw-Hill Book Company, 2001.

[2] Nakano, N., "Helical and Spiral Antennas - A Numerical Approach," Research Studies Press Ltd., John Wiley \& Sons Inc., 1987.

[3] Burke, G.J. \& Pogio, A.J., "NEC-2: Numerical Electromagnetics Code-Method of Moments," NOSC Technical Document 116, 1981.

[4] Kraus, JD, "A 50- Ohm Input Impedance for Helical Beam Antennas," IEEE Trans., AP-25 p. 913, Nov 1977.

[5] Kunio Sawaya., “ Antenna Design by Using Method of Moments," IEICE Trans., Comm., pp.1668-1677, Jan, 2005

[6] Can-Hui Chen, E.K.N. Yung, Bin-Jie Hu, Sheng-Li Xie ., "Axial mode helix antenna with exponential spacing ," Electronic Engineering Dept., City University of Hong Kong, China, 2007.

[7] Johnson, C.J. \& Jas., H., “Antenna Engineering Handbook," Second Edition, McGraw-Hill Book Company, 1984

[8] Sergey N. Makarov, "Antenna and EM Modeling with MATLAB ,"John Wiley \& Sons Inc, June, 2002 